\title{
RNA topology remolds electrostatic stabilization of viruses
}

\author{
Gonca Erdemci-Tandogan, ${ }_{1}^{1}$ Jef Wagner, ${ }^{1}$ Paul van der Schoot, ${ }^{2,3}$ Rudolf Podgornik, ${ }^{4,5}$ and Roya Zandi ${ }^{1}$ \\ ${ }^{1}$ Department of Physics and Astronomy, University of California, Riverside, California 92521, USA \\ ${ }^{2}$ Group Theory of Polymers and Soft Matter, Eindhoven University of Technology, P.O. Box 513, 5600 MB Eindhoven, The Netherlands \\ ${ }^{3}$ Institute for Theoretical Physics, Utrecht University, Leuvenlaan 4, 3584 CE Utrecht, The Netherlands \\ ${ }^{4}$ Department of Theoretical Physics, J. Stefan Institute, SI-1000 Ljubljana, Slovenia \\ ${ }^{5}$ Department of Physics, University of Ljubljana, SI-1000 Ljubljana, Slovenia
}

(Received 13 June 2013; published 14 March 2014)

\begin{abstract}
Simple RNA viruses efficiently encapsulate their genome into a nano-sized protein shell: the capsid. Spontaneous coassembly of the genome and the capsid proteins is driven predominantly by electrostatic interactions between the negatively charged RNA and the positively charged inner capsid wall. Using field theoretic formulation we show that the inherently branched RNA secondary structure allows viruses to maximize the amount of encapsulated genome and make assembly more efficient, allowing viral RNAs to out-compete cellular RNAs during replication in infected host cells.
\end{abstract}

DOI: 10.1103/PhysRevE.89.032707

PACS number(s): 87.15.-v, 41.20.Cv, 82.35.Rs, 87.14.gn

\section{INTRODUCTION}

Simple viruses encapsulate their genetic material into a protein shell, measuring no more than about $15 \mathrm{~nm}$ across for the smallest viruses and about $28 \mathrm{~nm}$ for a typical (plant) virus [1,2]. Under many circumstances, in vitro virus assembly is spontaneous and driven primarily by electrostatic interactions between negative charges on the backbone of the polynucleotide, usually single-stranded (ss) RNA, and positive charges on the virus coat proteins [3-10]. However, recent experiments indicate that RNA plays a role that goes beyond its polyelectrolyte (PE) nature, as some RNAs are encapsulated more efficiently than others [11]. For example, when viral RNA1 of BMV (brome mosaic virus) and CCMV (cowpea chlorotic mottle virus) are mixed in solution with the capsid proteins from CCMV, the BMV RNA is packaged three times more efficiently [11]. As the two RNAs differ in the amount of branching and their tertiary structure, both a straightforward consequence of their primary sequence, there must be a tight connection between capsid packing preferences and the structure of RNA [12,13].

In many viruses the number of negative charges on the ssRNA is larger than the number of positive charges on the virus coat proteins [14-17]. This overcharging phenomenon is intriguing and has been the subject of many papers. Belyi and Muthukumar examined a sample of actual viruses and found the ratio of the RNA charge to structural charge on the inner capsid surface to be around 1.6 [16]. While it seems to be feasible to encapsulate linear polymers with a number of charges as much as nine times that on the capsid proteins [18], which would result in strong "overcharging" of the virion, recent experiments show that the optimal number of charges residing on the linear PE is less than the total number of charges on the inner surface of viral shells [19], implying "undercharging" of the complex of capsid proteins with linear polyelectrolytes. This naturally leads to the question of which RNA features are implicated in the overcharging of the virion.

In what follows we show that RNA secondary structural features, such as branching, have a pronounced effect on the genome encapsulation capacity and thus could explain the phenomena of overcharging observed in viral particles.
In virtually all theoretical studies published to date investigating the overcharging in viruses, the genome is modeled as a simple linear polyelectrolyte chain [15,22-24]. Thus the phenomenon of overcharging is associated with many factors other than the structure of RNA [14-17,22]. Our numerical solutions of the polyelectrolyte Poisson-Boltzmann theory, without any additional assumptions regarding the effective monomer charge, do not support a universal overcharging of the virion. In fact, we find that mean-field PE theories for linear polymer chains lead to an undercharging phenomenon with fewer negative charges on the chain than positive ones on the capsid. This is consistent with several other recent numerical studies $[5,17,22]$. The overcharging in the viral particles has been explained through the genome-capsid $\mathrm{N}$-terminal interactions and/or Manning condensation [14-16,22]. However, it is important to note that the phenomenon of overcharging is also observed in viruses in which the number of charges on $\mathrm{N}$-terminals is not significant, e.g., dengue and yellow fever viruses [14].

While the theoretical studies of linear polymers shed some light on the overcharging phenomenon, recent experiments reveal the importance of RNA structure that goes beyond its polyelectrolyte nature as a linear charged chain $[11,12,25,26]$. Intrachain base paring, promoted by hydrogen bonding between mutually complementary nucleotides along the backbone, leads to a highly branched structure of the RNA molecule that furthermore promotes its compaction in free solution.

In this paper, we present a model that takes into account the combined effect of RNA branching and the genome-capsid protein electrostatic interactions. We find that the larger the inherent propensity to form branch points quantified by the fugacity, $f_{b}$, the larger is the optimal chain length that can be accommodated in the capsid, consistent with in vitro experiments. These results are evident from Fig. 1, showing the displacement in the position of the encapsulation free energy minimum towards longer chains as the branching fugacity increases. The inset to Fig. 1, showing the position of the minimum $N_{\min }$ as a function of the propensity for branching $f_{b}$, directly demonstrates this effect. 


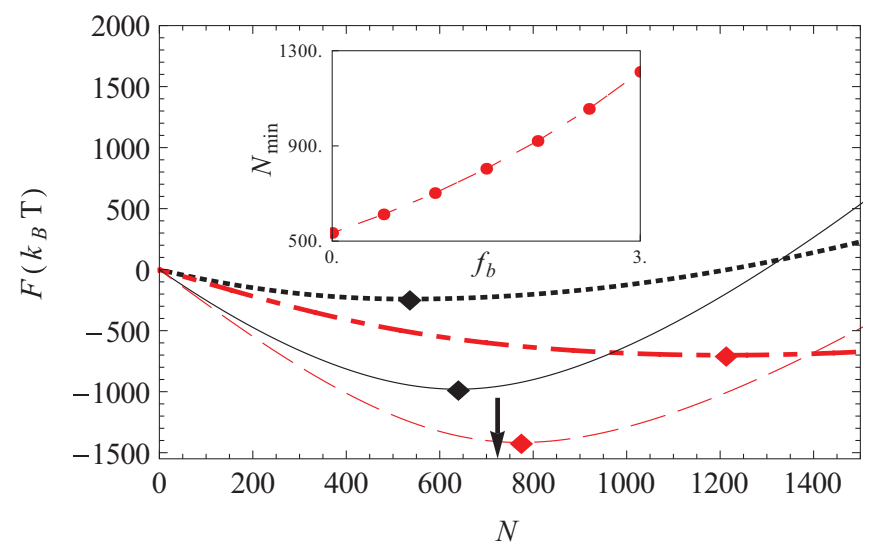

FIG. 1. (Color online) Encapsulation free energy as a function of monomer numbers for a linear polymer with $f_{b}=0$ (solid and dotted lines) and a branched polymer with $f_{b}=3$ (dashed and dotted-dashed lines) at two different values of $\mu$, corresponding to salt concentrations $10 \mathrm{mM}$ (solid and dashed lines) and $100 \mathrm{mM}$ (dotted and dotted-dashed lines). The arrow indicates the monomer number at which the full virus particle (capsid + polyelectrolyte) becomes neutral. Inset shows the position of the minimum $N_{\min }$ vs the branching fugacity $f_{b}$ for $100 \mathrm{mM}$ salt concentration. Other parameters used are $v=0.5, \tau=-1, \sigma=0.4, b=12$, and $T=$ $300 \mathrm{~K}$, typical for RNA and virus capsids $[2,15,20,21]$.

The figure also illustrates that the encapsulation free energy becomes more negative with increasing propensity of RNA to form branch points for a given number of monomers. This stabilization behavior suggests that branching is not only conducive to more efficient packing of the genome material into the virus shell, but also allows viral RNAs that have more branch points than other types of cellular RNAs [12] to out-compete the latter during replication in infected, susceptible host cells.

\section{THEORY}

To obtain the optimal length and the free energy associated with the encapsulated RNA inside a capsid, we model RNA as a generic flexible branched polyelectrolyte. Because of the physical character of the base pairing, the degree of branching of RNAs is statistical and may in the process of encapsulation be affected by interaction with the charges located on or near the inner surface of the protein coat. To this end, we consider only the case of annealed branched polymers in this paper [27].

Further, we consider that the RNA interacts with positive charges residing on the inner surface of a sphere, where for simplicity we additionally presume that the charges are not localized but smeared out uniformly. For a large proportion of viruses the positive charges are indeed located on the inner surface of the capsid that in reality is not a perfect sphere but has a structure on the nanometer scale [21]. For some viruses positively charged disordered domains on the coat proteins point into the capsid cavity in a brushlike fashion [14], a feature that we do not include in our coarse-grained model at this stage.

In the mean-field, ground-state approximation, the free energy of a negatively charged polymer chain confined to a positively charged, infinitely thin spherical shell can be written as

$$
\begin{aligned}
F= & \int d^{3} r\left\{\frac{1}{6}|\nabla \Psi(\mathbf{r})|^{2}+W[\Psi(\mathbf{r})]\right. \\
& \left.-\frac{1}{8 \pi \lambda_{B}}|\nabla \Phi(\mathbf{r})|^{2}-2 \mu \cosh [\Phi(\mathbf{r})]+\tau \Phi(\mathbf{r}) \Psi^{2}(\mathbf{r})\right\} \\
& +\int d^{2} r[\sigma \Phi(\mathbf{r})] .
\end{aligned}
$$

All quantities in Eq. (1) are dimensionless, so energies are in units of thermal energy $k_{B} T$ and lengths in units of the statistical step length (Kuhn length) of the polymer $a$. Here $\tau$ denotes the linear charge density of the polymer, $\sigma$ the surface charge density of the shell, $\Psi^{2}(\mathbf{r})$ the monomer density at position $\mathbf{r}$, and $\Phi(\mathbf{r})$ the mean electrostatic potential. The parameter $\mu$ is the fugacity (density) of the monovalent salt ions and corresponds to the concentration of salt ions in the bulk. The (dimensionless) Bjerrum length, $\lambda_{B}$, is a measure of the dielectric constant of the solvent, corresponding to about $0.7 \mathrm{~nm}$ for water at room temperature. The square gradient term in the first line of Eq. (1) describes the entropic cost for a nonuniform polymer density, and the last two lines in Eq. (1) describe the electrostatic interactions between the polyelectrolyte, the salt ions, and the charged capsid at the level of Poisson-Boltzmann theory [22]. The full derivation of the standard form of the free energy can be found in Refs. [28-32]. In addition to the standard form, we add the $W[\Psi]$ term that describes the statistics of an annealed branched polymer [33-36], given explicitly by

$$
W[\Psi]=\frac{1}{2} v \Psi^{4}-f_{e} \Psi-\frac{f_{b}}{6} \Psi^{3},
$$

where $v$ is the (dimensionless) excluded volume and $f_{e}$ and $f_{b}$ are the fugacities of the end and branch points, respectively.

In our description, the stem-loop or hair-pin configurations in RNA structures are counted as end points. The number of end and branch points $N_{e}$ and $N_{b}$ of the polymer depend on the fugacities $f_{e}$ and $f_{b}$ through

$$
N_{e}=-f_{e} \frac{\partial F}{\partial f_{e}} \quad \text { and } \quad N_{b}=-f_{b} \frac{\partial F}{\partial f_{b}} .
$$

Since we consider only the case of a single encapsulated polymer with no closed loops, there is a constraint on the number of end and branch points,

$$
N_{e}=N_{b}+2,
$$

with the degree of branching controlled by the fugacity of branch points $f_{b}$. The chain is linear if $f_{b}=0$ and becomes more branched as $f_{b}$ increases. The fugacity of end points $f_{e}$ is not a free parameter in the system, it is set through the above constraint, Eq. (4). In addition, the total number of polyelectrolyte monomers inside the capsid is fixed [37,38], i.e.,

$$
N=\int d^{3} r \Psi^{2}(\mathbf{r})
$$

which we enforce by introducing a Lagrange multiplier, $E$, when minimizing the free energy. 
We obtain the polyelectrolyte profile and electrostatic potential by varying the free energy functional with respect to fields $\Psi(r)$ and $\Phi(r)$ [39]. The resulting coupled set of nonlinear equations describes the monomer density field, $\Psi$, and the electrostatic potential $\Phi_{\text {in }}$, in the interior of the capsid, and the usual Poisson-Boltzmann equation for the electrostatic potential, $\Phi_{\text {out }}$, in the exterior of the capsid, viz.,

$$
\begin{aligned}
& \frac{1}{6} \nabla^{2} \Psi(\mathbf{r})=-E \Psi(\mathbf{r})+\tau \Phi_{\text {in }}(\mathbf{r}) \Psi(\mathbf{r})+\frac{1}{2} \frac{\partial W}{\partial \Psi} \\
& \nabla^{2} \Phi_{\text {in }}(\mathbf{r})=\frac{1}{\lambda_{D}^{2}} \sinh \left[\Phi_{\text {in }}(\mathbf{r})\right]-\frac{\tau}{2 \lambda_{D}^{2} \mu} \Psi^{2}(\mathbf{r}) \\
& \nabla^{2} \Phi_{\text {out }}(\mathbf{r})=\frac{1}{\lambda_{D}^{2}} \sinh \left[\Phi_{\text {out }}(\mathbf{r})\right]
\end{aligned}
$$

where $\lambda_{D}=1 / \sqrt{8 \pi \lambda_{B} \mu}$ is the (dimensionless) Debye screening length. The polymer segment concentration outside the capsid is assumed to be zero, $\Psi=0$. Equations (6) along with the constraints in Eqs. (4) and (5) represent a set of coupled nonlinear differential equations that, subject to appropriate boundary conditions, can only be solved numerically for the unknown parameters $f_{e}$ and $E$ and fields $\Psi$ and $\Phi$.

Assuming that the positive surface charge density, $\sigma$, is fixed, the electrostatic boundary condition (BC) is obtained by minimizing the free energy with respect to $\Phi$ on the surface, $\hat{n} \cdot \nabla \Phi_{\text {in }}-\hat{n} \cdot \nabla \Phi_{\text {out }}=4 \pi \lambda_{B} \sigma$. The choice of boundary conditions for $\Psi$ depends on how the polymer interacts with the capsid surface through nonelectrostatic forces. The strong short-ranged repulsion (as would be the case if we had included an excluded volume term between the polyelectrolyte monomers and the capsid proteins) leads to Dirichlet BCs. However, it turns out that for the large surface charge densities relevant to viruses, our conclusions are robust and do not depend on the choice of $\mathrm{BC}$; we come back to this below. In this paper, we focus on Neumann BCs, directly obtained from the minimization of the free energy in Eq. (1) with respect to the polymer density field, $\psi$, on the surface.

\section{RESULTS}

The overall dimensionless monomer density profiles $\mathcal{C}(r)=$ $\Psi(r)^{2}$ as a function $r=|\mathbf{r}|$, the distance from the center of the cavity, are shown in Fig. 2 for a linear polymer with $f_{b}=0$, and a branched polymer with $f_{b}=3.0$ of an equal number of segments, $N=1000$, enclosed in a spherical shell. The radius of the capsid is taken to be $b=12$ in units of the polymer Kuhn length that for our purpose is of the order of $1 \mathrm{~nm}$ [20]. Both types of polymers can adsorb onto the surface, and, interestingly, the branched polymer is adsorbed more strongly onto the surface than the linear chain.

We also investigated the spatial inhomogeneity in our annealed branched polymer model of RNA. In Fig. 2(a), we plot the dimensionless density of end points $C_{e}(r)=f_{e} \Psi(r)$ (solid line) and branches $C_{b}(r)=\frac{f_{b}}{6} \Psi^{3}(r)$ (dashed lines), obtained from Eq. (3). Figure 2(b) illustrates the fractions of end points $C_{e} / C$ (solid line) and fraction of branches $C_{b} / C$ (dashed lines) as a function of the distance from the center of the capsid. We observe that most branching takes place within a two Debye length layer, thus very near the capsid wall where the concentration of segments is high and the local gradient in

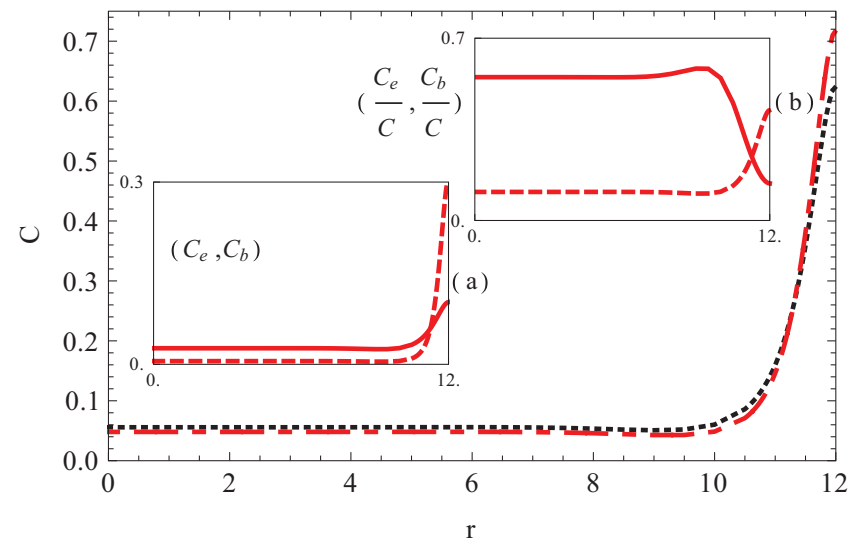

FIG. 2. (Color online) Concentration profile for $N=1000$ with $\mu$ corresponding to salt concentration $100 \mathrm{mM}$ and two different branching fugacities, $f_{b}=0$ (linear polymer) for the dotted line, and $f_{b}=3.0$ (branched polymer) for the dotted-dashed line. Inset (a) shows the concentration profile of end points (solid line) and branch points (dashed lines). Inset (b) shows the fraction of end points (solid line) and branch points (dashed lines). Other parameters used are $v=0.5, \tau=-1, \sigma=0.4, b=12$, and $T=300 \mathrm{~K}$.

density is the largest. This is straightforward to understand as branching increases the local density allowing more segments to interact with the wall. Figure 2 also shows that end points are dominantly distributed over the capsid interior. Thus branching can affect not only the segment distribution but also the structure of the adsorbed layer, making both quite nonuniform.

If we insert the parameter $f_{e}$ and fields $\Psi$ and $\Phi$ numerically obtained from Eqs. (4), (5), and (6) into Eq. (1), we can calculate the free energy of the chain-capsid complex, $F$. To obtain the encapsulation free energy using Eq. (1), we need to find the free energy difference between the chain-capsid complex and a free chain in solution and a positively charged capsid. The free energy of the free self-interacting chains (both linear and branched) is negligible under the stated conditions and is ignored. The capsid self-energy resulting from electrostatic interaction is calculated solving the system in the limit as $N \rightarrow 0$. Obviously, the capsid self-energy does not depend on the genome topology, but is not negligible and is explicitly subtracted. Here we emphasize that since capsid proteins spontaneously self-assemble in the absence of genome in different kind of viruses, we only focused on the free energy of complexation of chain-capsid interaction.

A plot of the encapsulation free energy $F$ vs the monomer number $N$, as shown in Fig. 1, confirms that the free energy minimum moves towards longer chains, i.e., allowing more monomers to be encapsulated into the viral shell. In the inset to Fig. 1, we plot the optimal polymer length $N_{\text {min }}$ (defined as the position of the free energy minimum) versus the branch point fugacity $f_{b}$ for $\mu$ corresponding to $100 \mathrm{mM}$. As illustrated in Fig. 1, this effect is more pronounced at high salt concentration.

For low salt concentrations, electrostatics overwhelms all the other interactions and branching becomes less pronounced, but it still has an effect so that a branched polymer is packaged more efficiently than a linear polymer. For instance, at $10 \mathrm{mM}$ salt the free energy has a minimum around $N=638$ for the linear polymer and $N=773$ for the branched polymer; see 
Fig. 1. At $100 \mathrm{mM}$ salt, the optimal number of monomers for the linear polymer is $N=534$, but for the branched polymer increases by more than two times to $N=1211$. The arrow on the $N$ axis of Fig. 1 corresponds to $N=4 \pi b^{2} \sigma$, representing a neutral system where the number of positive charges on the capsid wall is equal to the number of negative charges on the polymer chain. The aforementioned results correspond to the Neumann BC. As noted above, our conclusions do not depend on the type of BCs (Dirichlet vs Neumann) that we employed. For the Dirichlet BC, at $100 \mathrm{mM}$ salt, the optimal number of monomers is 202 for a linear polymer, and 930 for a branched polymer, consistent with the results for the Neumann BC.

This observation reveals that overcharging in viral particles could be a direct result of the secondary, i.e., branched, structure of viral RNA. We emphasize here that we repeated the above calculations for different surface charge densities, relevant to different viral capsids $(0.3 \leqslant \sigma \leqslant 0.9)$, and found that for all cases, the number of charges on linear polymers is less than the number of positive charges on the capsid. Quite interestingly, we also found that for any given linear charge density of the chain, the optimal length of encapsulated branched polymers is always larger than that of linear polymers.

Figure 1 also reveals the second important point, viz., that the free energies associated with branched polymers have deeper minima than those for linear polymers for a set of salt concentrations. This effect explains why some RNAs are encapsulated more efficiently than other RNAs, or other linear polyelectrolytes for that matter.

If coupling between RNA branching and electrostatics represents a robust mechanism, the details of its description should not be qualitatively important. To this end, it is interesting to compare our results for encapsulated charged branched polymer based on a field theoretic Ansatz for the statistics of branched polymers [33] with a very simple model with short-ranged attractive interaction between different chain segments mimicking the self-pairing of RNA bases [28]. We consider a linear polymer and now define the $W[\Psi]$ term as

$$
W[\Psi]=\frac{1}{2}(v-s w) \Psi^{4}+u \Psi^{6},
$$

with $s$ the average fraction of base pairs and $w$ the binding energy. We also include the next term in the virial expansion in order to stabilize the free energy since the total coefficient in front of the $\psi^{4}$ term can become negative. Calculating $F$ vs $N$ curves for increasing values of $s$, the average fraction of self-paired bases, we find the same qualitative behavior as for increasing branching fugacity: the position of the minimum moves towards longer polymers (larger $N$ ) and the depth of the minimum increases for increasing $s$. For example, at $10 \mathrm{mM}$ the free energy minimum is located at $N=632$ for $s=0$ and at $N=740$ for $s=0.04$. At $100 \mathrm{mM}$ salt the location of minimum moves from $N=524$ for $s=0$ to $N=903$ for $s=0.04$. Furthermore, as was the case for the branching model, the free energy minimum becomes deeper as the number of base pairs increases.

\section{DISCUSSION AND CONCLUSIONS}

Both models described above show that the total charge of the genome inside the capsid is larger than the one residing on the capsid interior and that the virion is thus overcharged. Our analysis clearly reveals that the genomic function of RNA, as encoded in its sequence that engenders its branched secondary structure, plays an important role in the self-assembly of ssRNA viruses. The branched secondary structure of RNA, treated with either branching or self-pairing models, promotes overcharging of the virion and stabilizes its equilibrium configuration. We emphasize that while our results differ from previous studies [17], a very recent coarse-grained molecular dynamics simulation of the assembly of viral particles completely confirms the importance of the structure of RNA in the assembly process [40]. Note that within our field theory formalism, we do not observe overcharging for linear chains. The condition of the solution and protein charge distribution in the simulations of Ref. [40] are such that overcharging could be observed for linear chains; nevertheless, polymer branching enhances overcharging, consistent with our studies.

In order to explain the experiments noted in the introduction on the competition between RNA of CCMV and BMV through the theory presented above, we calculated the number of branch points for both RNAs. In particular, we used RNASubopt, a program in the Vienna RNA package [41], to generate an ensemble of secondary structures for genome sequences of RNA1 of BMV and CCMV. We then calculated the thermally averaged number of branch points from the secondary structures of each RNA. We found that RNA1 of BMV has higher average number of branch points (65) than CCMV (60.5) confirming that in the absence of specific interactions RNA1 of BMV would be preferentially packaged over RNA1 of CCMV, consistent with the experimental results of Comas-Garcia et al. [11,42]. While one has to be cautious about results for longer sequences at high salt concentrations, the Vienna RNA Package [41] has been used to calculate thermally averaged properties of viral genomes with lengths of 2500-7000 nt, and important results have been obtained [12].

A comprehensive investigation of the physico-chemical parameters that impact capsid formation could have great potential in the development of antiviral therapies and a systematic understanding of the processes involved in viral infection.

\section{ACKNOWLEDGMENTS}

The authors would like to thank Mehran Kardar and Aaron Yoffe for useful discussions. This work was supported by the National Science Foundation through Grant No. DMR-1310687 (R.Z.).
[1] J. B. Bancroft, Adv. Virus Res. 16, 99 (1970).

[2] R. F. Bruinsma, Euro. Phys. J. E 19, 303 (2006).

[3] F. D. Sikkema, M. Comellas-Aragones, R. G. Fokkink, B. J. M. Verduin, J. Cornelissen, and R. J. M. Nolte, Org. Biomol. Chem. 5, 54 (2007).
[4] Y. P. Ren, S. M. Wong, and L. Y. Lim, J. Gen. Virol. 87, 2749 (2006).

[5] P. Ni, Z. Wang, X. Ma, N. C. Das, P. Sokol, W. Chiu, B. Dragnea, M. Hagan, and C. C. Kao, J. Mol. Biol. 419, 284 (2012). 
[6] P. van der Schoot and R. Zandi, Phys. Biol. 4, 296 (2007).

[7] A. Losdorfer Bozic, A. Siber, and R. Podgornik, J. Biol. Phys. 39, 215 (2013).

[8] M. C. Daniel, I. B. Tsvetkova, Z. T. Quinkert, A. Murali, M. De, V. M. Rotello, C. C. Kao, and B. Dragnea, ACS Nano 4, 3853 (2010).

[9] A. Zlotnick, R. Aldrich, J. M. Johnson, P. Ceres, and M. J. Young, Virology 277, 450 (2000).

[10] H.-K. Lin, P. van der Schoot, and R. Zandi, Phys. Biol. 9, 066004 (2012).

[11] M. Comas-Garcia, R. D. Cadena-Nava, A. L. N. Rao, C. M. Knobler, and W. M. Gelbart, J. Virol. 86, 12271 (2012).

[12] A. M. Yoffe, P. Prinsen, A. Gopal, C. M. Knobler, W. M. Gelbart, and A. Ben-Shaul, Pub. Natl. Acad. Sci. USA 105, 16153 (2008).

[13] F. Li Tai, W. M. Gelbart, and A. Ben-Shaul, J. Chem. Phys. 135, 155105 (2011).

[14] T. Hu, R. Zhang, and B. I. Shklovskii, Physica A 387, 3059 (2008).

[15] P. van der Schoot and R. Bruinsma, Phys. Rev. E 71, 061928 (2005).

[16] V. A. Belyi and M. Muthukumar, Pub. Natl. Aacad. Sci. USA 103, 17174 (2006)

[17] C. L. Ting, J. Z. Wu, and Z. G. Wang, Pub. Natl. Aacad. Sci. USA 108, 16986 (2011).

[18] Y. F. Hu, R. Zandi, A. Anavitarte, C. M. Knobler, and W. M. Gelbart, Biophys. J. 94, 1428 (2008).

[19] R. D. Cadena-Nava, Y. F. Hu, R. F. Garmann, B. Ng, A. N. Zelikin, C. M. Knobler, and W. M. Gelbart, J. Phys. Chem. B 115, 2386 (2011).

[20] R. Zandi and P. van der Schoot, Biophys. J. 96, 9 (2009).

[21] A. L. Bozic, A. Siber, and R. Podgornik, J. Biol. Phys. 38, 657 (2012).

[22] A. Siber and R. Podgornik, Phys. Rev. E 78, 051915 (2008).

[23] O. M. Elrad and M. F. Hagan, Phys. Biol. 7, 045003 (2010).
[24] M. F. Hagan, Adv. Chem. Phys. 155, 1 (2014).

[25] A. Borodavka, R. Tuma, and P. G. Stockley, Pub. Natl. Acad. Sci. USA 109, 15769 (2012).

[26] P. G. Stockley, R. Twarock, S. E. Bakker, A. M. Barker, A. Borodavka, E. Dykeman, R. J. Ford, A. R. Pearson, S. E. V. Phillips, N. A. Ranson et al., J. Biol. Phys. 39, 277 (2013).

[27] A. McPherson, BioEssays 27, 447 (2005).

[28] I. Borukhov, D. Andelman, and H. Orland, Euro. Phys. J. B 5, 869 (1998).

[29] I. Borukhov, D. Andelman, and H. Orland, Europhys. Lett. 32, 499 (1995).

[30] I. Borukhov and D. Andelman, Macromolecules 31, 1665 (1998).

[31] I. Borukhov, D. Andelman, and H. Orland, J. Phys. Chem. B 103, 5042 (1999).

[32] A. Shafir, D. Andelman, and R. R. Netz, J. Chem. Phys. 119, 2355 (2003)

[33] T. C. Lubensky and J. Isaacson, Phys. Rev. A 20, 2130 (1979).

[34] T. T. Nguyen and R. F. Bruinsma, Phys. Rev. Lett. 97, 108102 (2006).

[35] S. I. Lee and T. T. Nguyen, Phys. Rev. Lett. 100, 198102 (2008).

[36] K. Elleuch, F. Lequeux, and P. Pfeuty, J. Phys. I France 5, 465 (1995).

[37] P.-G. de Gennes, Macromolecules 15, 492 (1982).

[38] H. Ji and D. Hone, Macromolecules 21, 2600 (1988).

[39] A. Siber, R. Zandi, and R. Podgornik, Phys. Rev. E 81, 051919 (2010).

[40] J. D. Perlmutter, C. Qiao, and M. F. Hagan, eLife 2, e00632 (2013).

[41] I. L. Hofacker, W. Fontana, P. F. Stadler, L. S. Bonhoeffer, M. Tacker, and P. Schuster, Monatsh. Chem. 125, 167 (1994).

[42] The standard deviation for the number of branch points in the ensemble of RNA secondary structures is around 2.5 for the RNA1 sequence of both BMV and CCMV. 